\title{
COMPARATIVE STUDY OF THE GRID-SCALE AND SUBGRID-SCALE VELOCITY FIELDS - A PRIORI TEST
}

\author{
M. Ashraf Uddin ${ }^{1}$, M. Matiar Rahman ${ }^{1}$ and M. Saiful Islam Mallik ${ }^{2}$ \\ ${ }^{1}$ Department of Mathematics \\ Shahjalal University of Science \& Technology, Sylhet, Bangladesh \\ ${ }^{2}$ Department of Arts and Science \\ Ahsanullah University of Science \& Technology, Dhaka, Bangladesh \\ E-mail: auddin-mat@sust.edu
}

Received 17.05.08 Accepted 19.06.10

\begin{abstract}
Generation of grid-scale (GS) and subgrid-scale (SGS) velocity fields is performed by direct filtering of DNS (Direct Numerical Simulation) data at a low Reynolds number in homogeneous isotropic turbulence in order to assess the spectral accuracy as well as the performance of filter functions for LES (Large Eddy Simulation). The filtering is performed using three classical filter functions: Gaussian, Tophat and Sharp cutoff filters and in all three cases the results are compared with three different filter widths for LES. Comparing the distributions of GS and SGS velocities, and the decay of turbulence with those from DNS fields through out the whole calculation we have found that among the three filter functions, the performance of Sharp cutoff filter is better than that of the other two filter functions in terms of both spatial spectra and the distribution of velocities. Furthermore, it is shown that the accuracy of the filtering approach does not depend only on the filter functions but also on the filter widths for LES.
\end{abstract}

\section{Introduction}

Direct numerical simulation (DNS) and large-eddy simulation (LES) have been widely used to study the physics of turbulence (Moin and Kim [1]; Viecelli [2]; Kato and Ikegawa [3]; Kato et al. [4]; Uddin et al. [5]). Nowadays, these numerical techniques are considering as the accurate and sophisticated predictive methods for flows of engineering interest. The DNS is considered as the exact approach to turbulence simulation but it is too expensive, and is only possible for simple and low Reynolds number flows. The recent development of supercomputers enabled to carry out the DNS of Navier-Stokes equations, and to explain the statistical properties and organized structures of turbulence for relatively high Reynolds number flows (Vincent and Meneguzzi [6]; Jimenez et al. [7]; Tanahashi et al. [8]; Tanahashi et al. [9]; Uddin et al. [10]) but the grid dependence is very high (proportional to $R e^{9 / 4}$ ) and the calculation is fairly time consuming, so that the DNS is not appropriate to the practical use. Generally, industrial, natural or experimental configurations involve Reynolds numbers that are far too large to allow direct numerical simulation, and in these flows the only possible method is large-eddy simulation. The LES is less expensive and can simulate very complex flow fields in turbulence. In LES method, large-scale motion is exactly calculated and the effect of subgrid-scale (SGS) 
motions on the evolution of large scales, which is expected to be universal, is modeled. The characteristic of the LES calculation is fully three-dimensional and unsteady. Therefore, to model complex flow configurations as well as for engineering applications, the use of LES is becoming increasingly common day by day.

The scale selection that the large-eddy simulation technique is based on a separation between large and small scales (Sagaut [11]). In order to define these two categories, a reference or cutoff length first has to be determined. Those scales that are of a characteristic size greater than the cutoff length are called large or resolved scales or gridscales, and others are called small or subgrid scales. The latter are included by way of a statistical model called a subgrid-scale (SGS) model.

For SGS modeling, the most commonly used SGS model is Smagorinsky eddy viscosity model (Smagorinsky [12]). Because of growing popularity of LES, recent research has been aimed at developing robust LES models, such as dynamic subgrid scale model (Germano et al. [13]), isotropic eddy viscosity model (Yoshizawa [14]), etc. and these models are derived based on some assumption about the nature of the subgrid turbulence. However, all of the models have some defect in itself, and still today, people are doing their efforts to develop new and accurate SGS model for LES. Concerning the subgridscale model it seems quite important to know what happened in the filtered field for LES.

The governing equation for LES is the filtered Navier- Stokes equations, so that we need to filter the Navier-Stokes equations with effective filter functions. There are several filter functions that are used to filter the Navier-Stokes equations, and using these filterfunctions we can decompose the velocity fields into grid-scale and subgrid-scale velocities. Therefore, to develop the SGS model for LES, it is very important to study the behavior of turbulence in the filtered velocity fields. Since the DNS is considered as the exact solution of Navier-Stokes equations, so it would be effective to know the features of grid-scale and subgrid-scale turbulence by direct filtering of DNS data.

Therefore, the objective of this study is to generate the grid-scale (GS) and subgrid-scale (SGS) velocity fields by direct filtering of the DNS data using three classical filter functions in LES. We compare the behavior of the velocity distributions as well as the decay of turbulence in the GS and SGS fields with the results in the DNS data with $R e_{\lambda}$ $=30.5$ to perform $a$ priori test in homogeneous isotropic turbulence. The filter width plays very important role in this filtering process. Hence, the over all goal of this study is to show the accuracy of the filtering approach as well as to show the performance of the different filter functions with several filter widths.

\section{Grid-scale and Subgrid-scale Velocity Fields}

\subsection{DNS data base}

The reference DNS is performed at $64^{3}$ resolutions by using a spectral code (Tanahashi et al. [8]; Tanahashi et al. [9]; Uddin et al. [10]) and the computation was done with nondimensional $\Delta t=0.00316$. At the end of calculation, the Reynolds number, $R e_{\lambda}$, based on $u_{\mathrm{rms}}$ and Taylor microscale $(\lambda)$ of the DNS data is $30.5(t=3.792)$ and the maximum possible Reynolds number of the flow is 121.1. A sample of mesh in the periodic box is 
shown in Fig. 1.

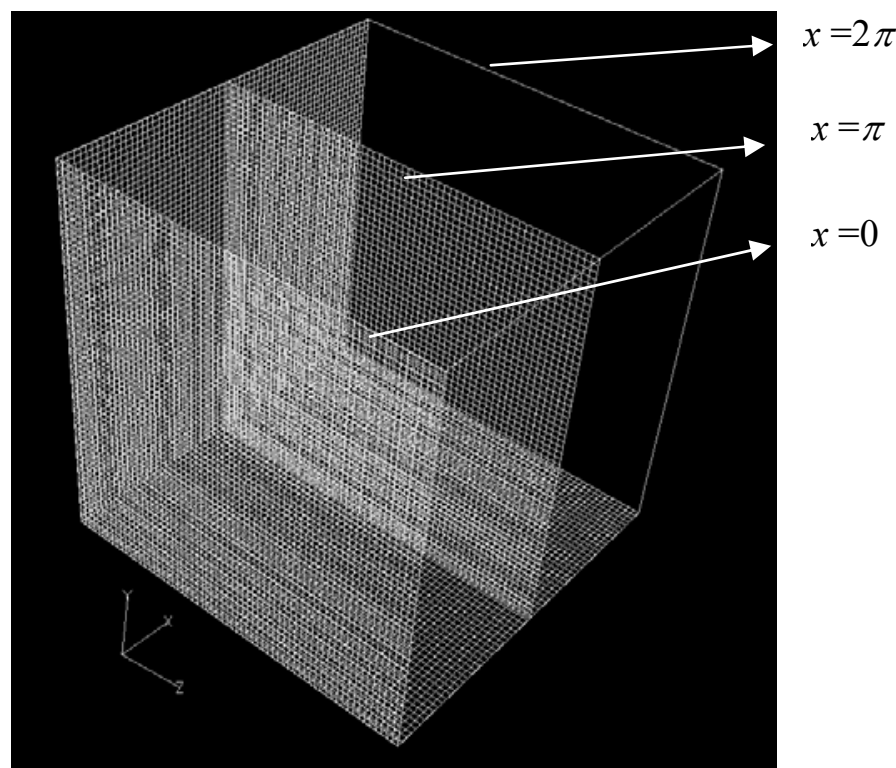

Figure 1: A sample of mesh generation in the periodic box. Computational domain: $2 \pi \times 2 \pi \times 2 \pi$; Grid points: $64 \times 64 \times 64$.

\subsection{Classical filters for LES}

In this study, three classical filters are used for performing the spatial scale separation. The filtering of DNS data is carried out in the Fourier space rather than in the physical space. For a filtered width $\Delta_{\mathrm{i}}$ in $i$-direction, these filter functions in Fourier space are given as follows:

(i) Gaussian filter:

$$
\hat{G}\left(k_{i}\right)=\exp \left\{-\left(\Delta_{i} k_{i}\right)^{2} / 24\right\}
$$

(ii) Tophat filter:

$$
\hat{G}\left(k_{i}\right)=\sin \left(\frac{\Delta_{i} k_{i}}{2}\right) /\left(\frac{\Delta_{i} k_{i}}{2}\right)
$$

(iii) Sharp cutoff filter:

$$
\hat{G}\left(k_{i}\right)= \begin{cases}1, & \left(\left|k_{i}\right| \leq \frac{\pi}{\Delta_{i}}\right) \\ 0, & \left(\left|k_{i}\right|>\frac{\pi}{\Delta_{i}}\right)\end{cases}
$$




\subsection{Generation of GS and SGS velocity fields}

To generate the grid-scale (GS) and subgrid scale (SGS) velocity fields, we have directly filtered the DNS velocity fields using above three classical filters: Gaussian filter, Tophat filter and Sharp-cutoff filter for LES. In LES, a velocity component $u$ can be decomposed into two components, one component is in the range of low wave-number of energy spectrum, called GS component and is denoted by $\bar{u}$, and the other component is in the range of high wave-number of energy spectrum, called SGS component and is denoted by $u^{\prime}$. Their relation can be expressed as:

$$
u=\bar{u}+u^{\prime}
$$

The filtering is represented mathematically in physical space as a convolution product (Leonard [15]). The filtered part $\bar{u}$ of the variable $u$ is defined formally by the relation:

$$
\bar{u}(x)=\int_{D} G\left(x-x^{\prime}, \Delta\right) u\left(x^{\prime}\right) d x^{\prime},
$$

in which $D$ is the entire domain, $G$ is filter kernel function and $\Delta$ is the filter width.

The dual definition in the Fourier space can be obtained by multiplying the spectrum $\hat{u}(\mathbf{k})$ of $u(x)$ by the spectrum $\hat{G}(\mathbf{k})$ of the kernel $G(\mathrm{x})$ such that,

$$
\hat{\bar{u}}(\mathbf{k})=\hat{G}(\mathbf{k}) \hat{u}(\mathbf{k}), \mathbf{k}=0, \pm 1, \pm 2, \ldots \ldots \ldots
$$

The function $\hat{G}$ is the transfer function associated with the kernel $G$.

Using three filters functions given in section 2.2 for LES in the Fourier space, one set of DNS data for $R e_{\lambda}=30.5$ is filtered and the exact GS velocity fields, $\bar{u}$ are obtained. After generating $\bar{u}$, the SGS velocity field can be obtained by computing the relation:

$$
u^{\prime}=u-\bar{u}
$$

Filter width plays very important role with filter functions in this process. In LES calculation, the characteristic filter width $\Delta_{\mathrm{i}}$ is commonly used as the length, approximately proportional to the grid interval, $\Delta x=2 \pi / N$, where $N$ is the number of grid points in any direction (Piomelli [16]; Horiuti [17]). The structures represented by the GS and SGS velocities consequently depend both on the grid interval and on the type of filter employed. In this study, we are interested to discuss the behaviors of filtered DNS velocity fields by using three classical filters for LES. Therefore, three different filter width $\Delta_{i}=2 \Delta x, 4 \Delta x$ and $8 \Delta x$ are considered for all three classical filters, where $\Delta x$ is the grid distance in DNS calculation. Since, we are dealing with homogeneous isotropic turbulence, the filter width $\Delta_{i}$ is same in each direction and hereafter it is denoted by $\bar{\Delta}$.

\section{Results and Discussions}

\subsection{Distributions of the DNS, GS and SGS velocities}

For any integer $N>0$, the set of points $x_{j}=2 \pi j / N, j=0,1, \ldots \ldots \ldots, N-1$, in any direction 
referred to as nodes or grids, where $N$ is the number of grid points in that direction. In order to understand the GS velocity field from the DNS velocity field, we have compared the filtered velocity with DNS velocity using several filter widths. For this purpose we have randomly chosen one-dimensional velocity profile, say at $x_{1}$, and have plotted $u_{1}\left(x_{1}\right)$ and $\bar{u}_{1}\left(x_{1}\right)$ for $R e_{\lambda}=30.5$ in Figs. 2(a)-2(c) using three different filter widths, $\bar{\Delta}=2 \Delta x, 4 \Delta x$ and $8 \Delta x$ respectively, where $\Delta x=2 \pi / N$.

In all cases, we have obtained these one dimensional profiles using the three filter functions. The profiles in Fig. 2(a), show that the GS velocity fields with $\bar{\Delta}=2 \Delta x$ for all three filter functions are very close to the DNS velocity field through out the whole analysis.

Figure 2(b) revealed that the profiles of GS velocity for all three filter functions with $\bar{\Delta}=$ $4 \Delta x$ do not collapse with the DNS velocity field through out the whole analysis. Here in the lower and higher grid ranges GS velocity profiles under-estimate the DNS profile, but at the middle stage the GS velocity profiles over-estimates the DNS profile. Clearly we can see that the separation of GS velocity with sharp-cutoff filter seems to be better than that of the other two filter functions. The GS velocity with filter width $\bar{\Delta}=8 \Delta x$ is highly separated from the DNS for all three filter functions in Fig. 2(c), but in this case the GS velocity has less contribution over the whole computed flow fields that will be shown in the later section.

Therefore, from Figs. 2(a)-2(c) clearly we can observe that although for all three filter width the DNS velocity field can be separated but the filter width $\bar{\Delta}=4 \Delta x$ shows significant results for this Reynolds number case for all three filter functions, but with $\bar{\Delta}=8 \Delta x$ most of the velocity fields accumulate to the SGS part that will be shown later. Moreover, among the three filter functions the Sharp cutoff filter shows better result than that of the other two filter functions. This suggests that accuracy of the filtering depends on the filter functions as well as on the filter width of the computation.

If we choose the one-dimensional velocity profile, say at $y_{1}$ or $z_{1}$, we get nearly the similar results for all three filter functions as well as for all three filter widths. 

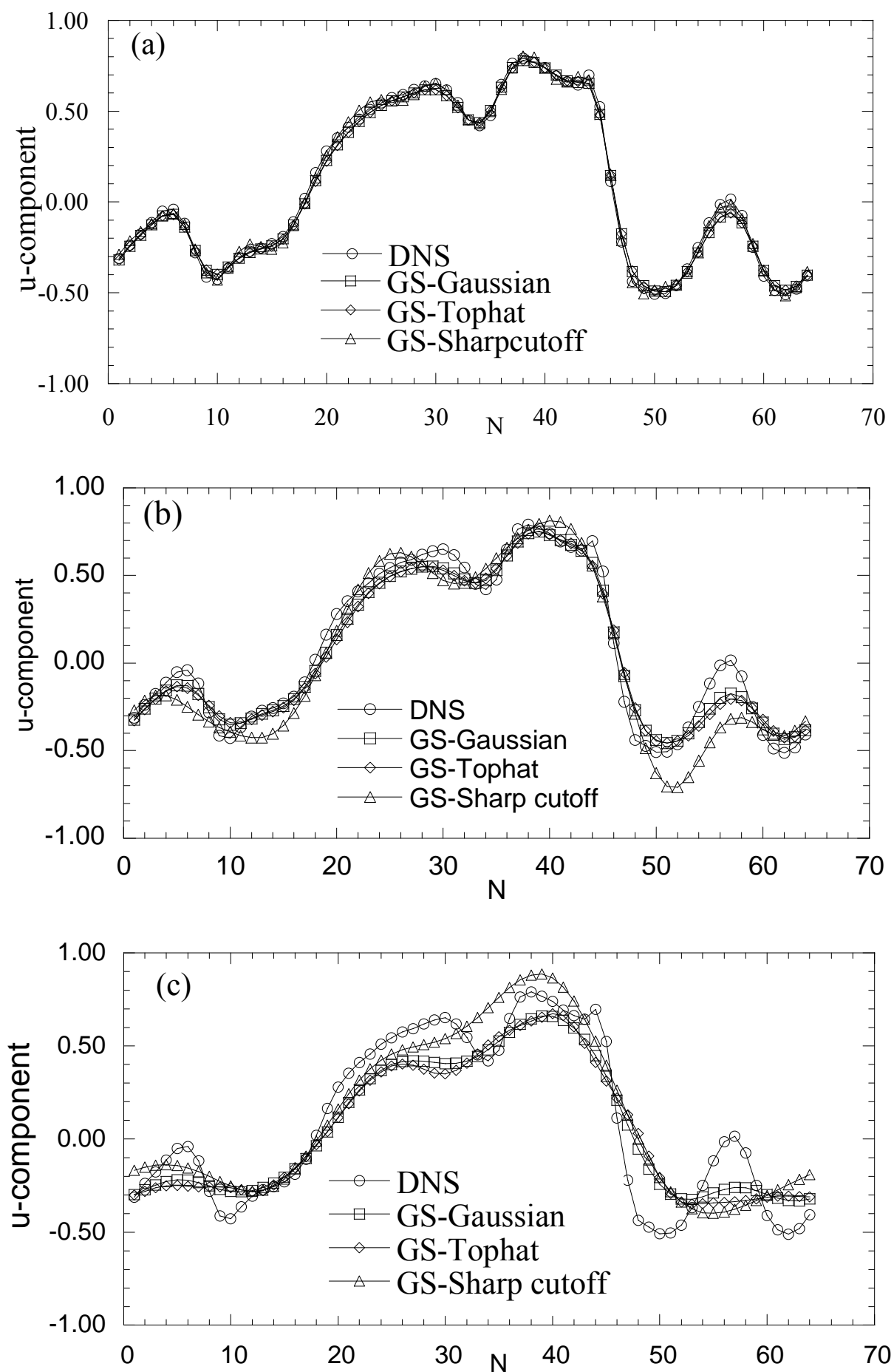

Figure 2: Comparison of u-component in DNS and GS velocity fields for three filter functions with three filter widths, $\bar{\Delta}=$ (a) $2 \Delta \mathrm{x}$; (b) $4 \Delta \mathrm{x}$ and (c) $8 \Delta \mathrm{x}$. 

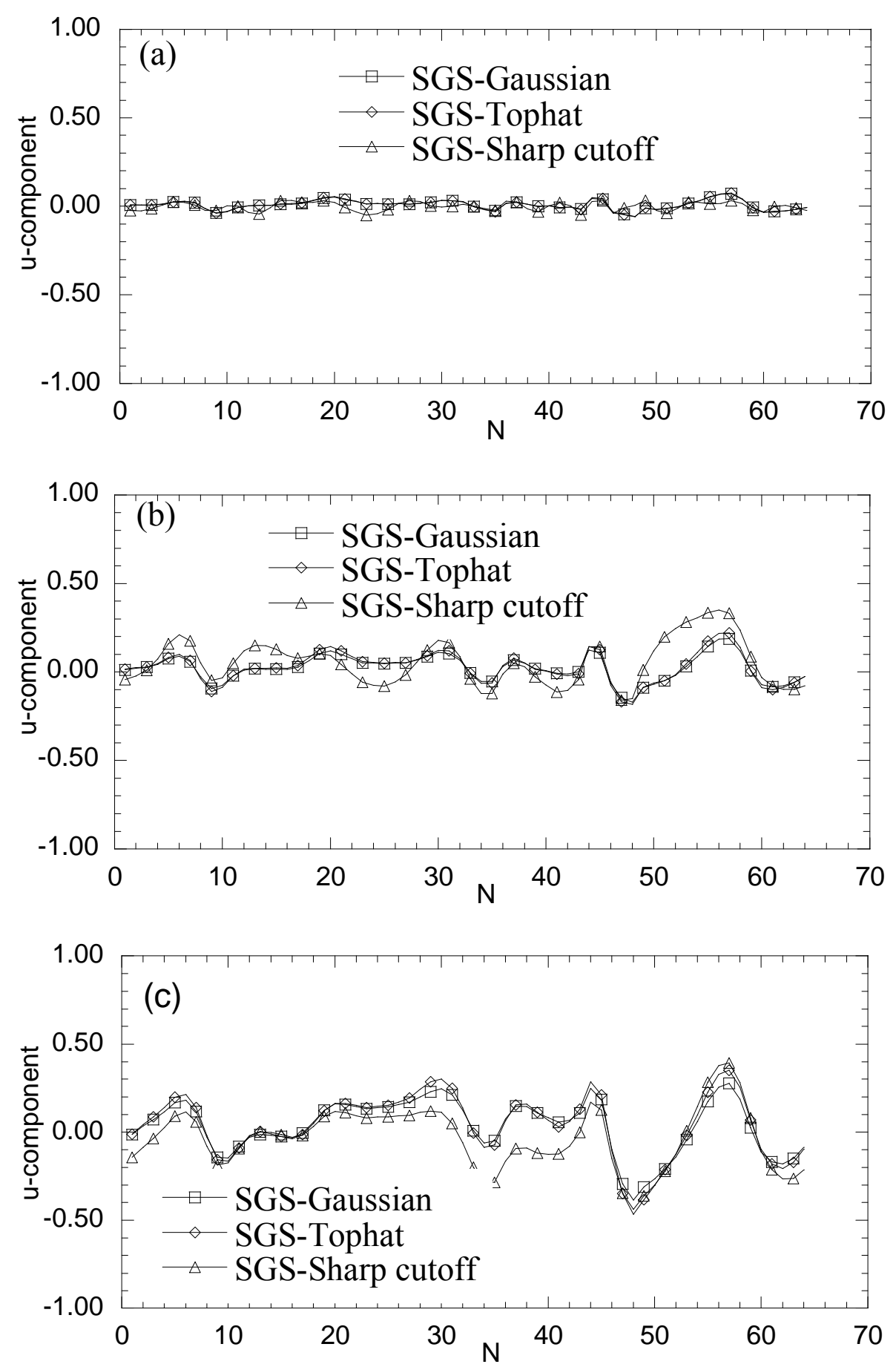

Figure 3: Comparison of u-component in SGS velocity fields for the three filter functions with the filter widths, $\bar{\Delta}=$ (a) $2 \Delta \mathrm{x}$; (b) $4 \Delta \mathrm{x}$ and (c) $8 \Delta \mathrm{x}$.. 
The comparisons of the SGS velocity fields for all three filter functions with different filter widths are shown in order to understand the flow pattern and its development in the whole analysis. Figs. 3(a)-3(c) represent the one-dimensional SGS velocity profile, $u_{1}^{\prime}\left(x_{1}\right)$ for all three filter widths and three filter functions. The profiles in Fig. 3(a) show that the SGS velocity fields with $\bar{\Delta}=2 \Delta x$ for all three filter functions are close to each other through out the whole analysis. In Fig. 3(b) for $\bar{\Delta}=4 \Delta x$, we observe that the SGS velocity fields for two filter functions: Gaussian and Tophat filter collapsed to each other, but the results for Sharp cutoff filter function is different from that of given by the other two filter functions. The development of the flow fields also suggest that the separation of SGS fields for this filter width is better than that of given in Fig. 3(a). Here also it is important to note that the contribution of the SGS fields for $\bar{\Delta}=4 \Delta x$ is significant. But, in

Fig. 3(c) for the filter width $\bar{\Delta}=8 \Delta x$, the contribution of the SGS velocity fields is more larger than that of the GS velocity fields that will be shown later.

\subsection{Decay of turbulence in DNS, GS and SGS fields}

Three-dimensional energy spectra in DNS and GS velocity fields for all three filter functions with three filter widths $\bar{\Delta}=2 \Delta x, 4 \Delta x$ and $8 \Delta x$ are presented in Fig.4, in which energy spectrum is calculated by the definition given as follows:

$$
E(k)=\sum_{k-\frac{1}{2}<|k| \leq k+\frac{1}{2}} \frac{1}{2} \hat{u}(\mathbf{k}) \hat{u}^{*}(\mathbf{k})
$$

where $\boldsymbol{k}$ is the wave number vector. Here, the GS spectra are obtained by using Gaussian, Tophat and Sharp-cutoff filters and then compared to the DNS spectrum. The DNS spectrum for this $R e_{\lambda}$ case shows the power decay close to $k^{-5 / 3}$. Here in all figures, the abscissa and the ordinate of the coordinate system represent the wave number $|k|$ and energy spectra $E(k)$, respectively. In Fig. 4(a) for $\bar{\Delta}=2 \Delta x$, we see that the GS fields contain the velocity in a full range in case of the Gaussian and Tophat filters. But with the Sharp-cutoff filter the GS spectrum does not contain the velocity in a full wave number range.

Since Sharp cutoff filter strictly cut or separate the wave number-range (grids in physical space) depending on the filter width, hence the flow field is exactly separated into two parts such as GS and SGS part. So we can see that the three dimensional energy spectrum in GS field for Sharp-cutoff filter exactly collapsed with DNS spectrum from lowest wave number to the cutoff wave number. This behavior also confirms the accuracy of the filtering process. 

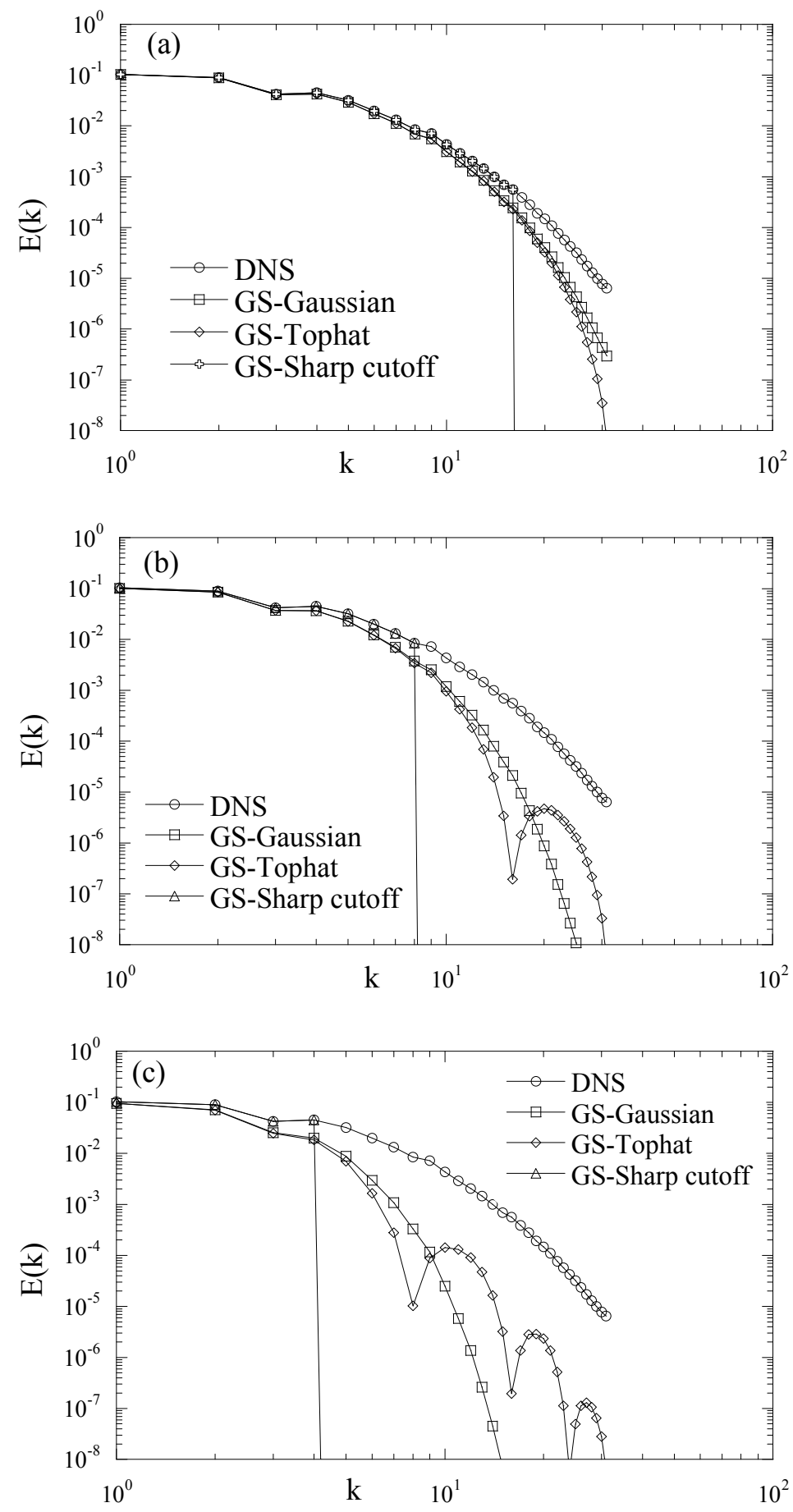

Figure 4: Comparison of three-dimensional energy spectra in DNS and GS velocity fields for three filter functions with three filter widths, $\bar{\Delta}=$ (a) $2 \Delta \mathrm{x}$; (b) $4 \Delta \mathrm{x}$ and (c) $8 \Delta \mathrm{x}$. 
In Figs. 4(b) and 4(c), the profiles of energy spectra for all three filter functions and different filter widths show the similar pattern as shown in Fig. 4(a). In both cases the GS spectrum for the Sharp-cutoff filter function does not contain the velocity in a full wave number range. Here we note that in the high wave number range the energy spectra for Tophat filter show some fluctuation or irregular behavior and this fluctuation repeated several times for the largest filter width. It may happen due to the nonlinear interaction or the limitation of the Tophat function itself but the exact reason is unclear. Whatever it is, the contribution of the energy spectra reduces with the increase of filter width for all three filter functions, and like the distribution of one-dimensional velocities given in the previous section, we can also say that the filter width $\bar{\Delta}=4 \Delta x$ gives good results in this filtering approach for this Reynolds number case.

Figures 5(a)-5(c) represent the three dimensional energy spectra in DNS and SGS velocity fields. In this case the energy spectra in SGS fields are also calculated using the same definition given in Eqn. (8). Here the SGS spectra are also obtained by using the Gaussian, Tophat and Sharp-cutoff filter functions, and then compared to the DNS spectrum.

Form these figures clearly we can observe that the whole wave number range contributes on the energy spectra in SGS velocity fields in case of Gaussian and Tophat filter functions. On the other hand, only the cutoff wave numbers contribute on the energy spectra in case of Sharp cutoff filter. Therefore, in Fig. 5(a) we see that the decay of energy spectrum in SGS field for Sharp cutoff filter exactly collapsed with DNS spectrum in the high wave number range, and the contribution of this spectrum in the filtered field is not so high for the filter width $\bar{\Delta}=2 \Delta x$. Like GS field the profile of energy spectrum for Sharp cutoff filter again confirms the accuracy of this filtering approach.

In Fig. 5(b), for $\bar{\Delta}=4 \Delta x$, the profiles of energy spectrum for all three filter functions show the similar pattern as shown in Fig. 5(a). But, in this case, the cutoff wave numbers contribute significantly on the energy spectra in case of Sharp cutoff filter. Here, we can say that for the filter width $\bar{\Delta}=4 \Delta x$, the contribution of this spectrum in the filtered field is reasonable for all three filter functions. This behavior also matches with the results given in Fig. 4(b). That is the filter width $\bar{\Delta}=4 \Delta x$ separate the DNS velocity field into GS and SGS fields reasonably for all three filter functions used in this study.

Finally, in Fig. 5(c) for $\bar{\Delta}=8 \Delta x$, although we get the similar pattern like in figures 5(a) and 5(b), but in this case we observe that almost whole flow field goes into the SGS field. That is, for this filter width case the SGS field is very much similar to the DNS field, which we also realized from the results given in Fig. 4(c). Whatever it is, the decay of turbulence given in Figs. 5(a)-5(c) revealed that the contribution of the energy spectra in SGS fields increases with the increase of the filter width for all three filter functions. 

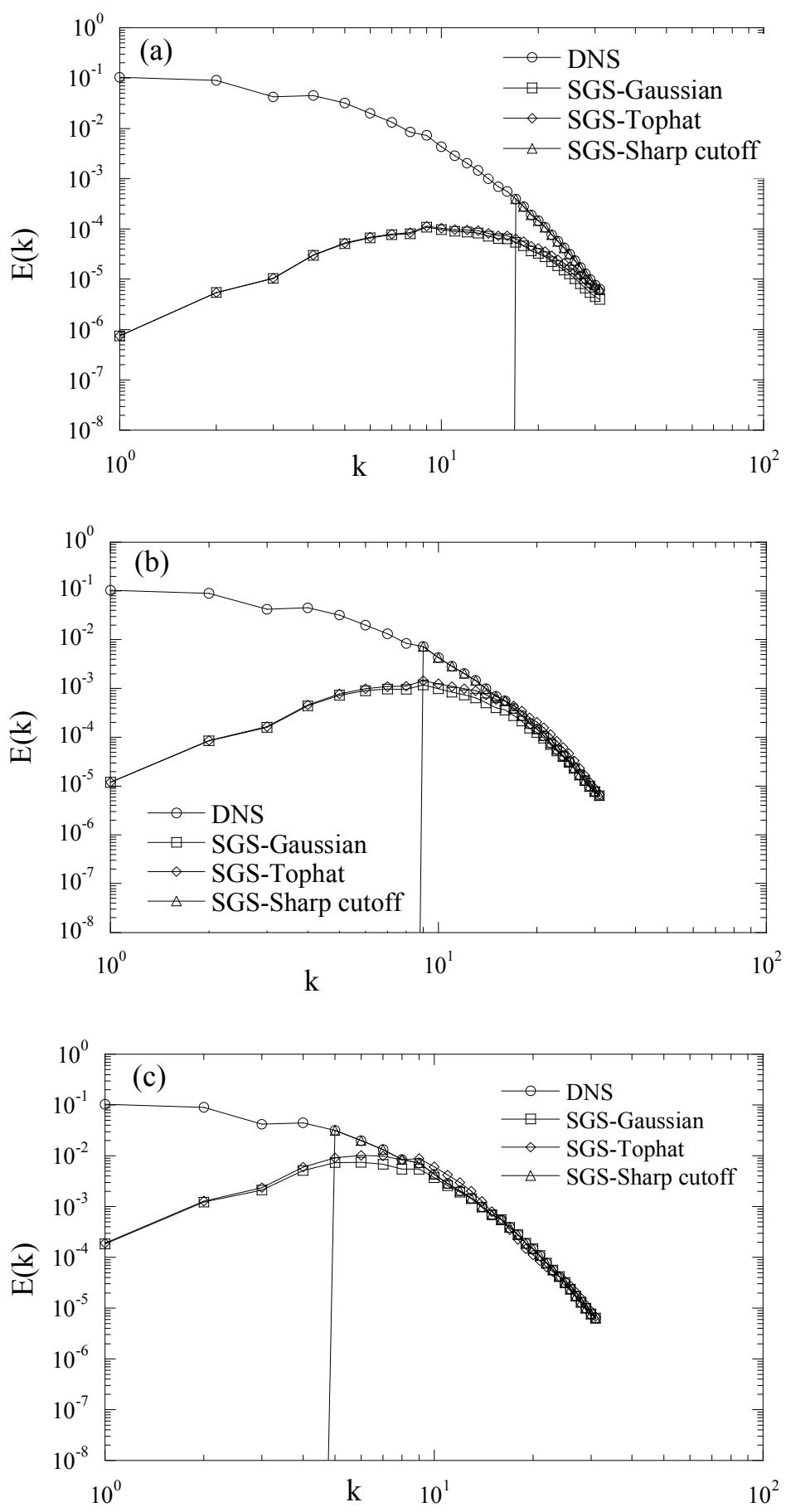

Figure 5: Comparison of three-dimensional energy spectra in DNS and SGS velocity fields for three filter functions with the three filter widths, $\bar{\Delta}=$ (a) $2 \Delta x$; (b) $4 \Delta x$ and (c) $8 \Delta x$.. 


\section{Conclusions}

The conclusion of this study can be drawn as follows:

We have successfully filtered the DNS fields in homogeneous isotropic turbulence at a low Reynolds number $\left(R e_{\lambda}=30.5\right)$ with $64^{3}$ grids and generate the grid scale (GS) and subgrid scale (SGS) fields, using three classical filters and three different filter widths for LES. Then we have compared the behavior of the one-dimensional velocity fields as well as the three-dimensional energy spectra in the GS and SGS fields with the results in the DNS field. After analyzing the results of velocity distributions and the decay of turbulence in all DNS, GS and SGS fields through out the whole calculation we have found that among the three filter functions: Gaussian, Tophat and Sharp cutoff filters, the performance of Sharp cutoff filter is better than that of the other two filter functions for this filtering approach. It is revealed that the results with Gaussian filter are also good but the performance of the Tophat filter is the least. It is also shown that among three filter widths, such as $\bar{\Delta}=2 \Delta x, 4 \Delta x$ and $8 \Delta x$, the GS and SGS results with the filter width $\bar{\Delta}=$ $4 \Delta x$ are more reasonable for all three filter functions for this Reynolds number case. Hence, our present study suggests that the accuracy of the filtering process depends on the filter functions as well as on the filter widths for LES.

\section{ACKNOWLEDGEMENT}

The main computation of DNS and the filtering were carried out on a large-scale massively parallel supercomputer, Hitachi SR8000, located at the University of Tokyo using secure remote access through the networking system of Shahjalal University of Science \& Technology. We gratefully acknowledge their support and cooperation for this computation.

\section{REFERENCES}

1. Moin, P and Kim, J., Numerical Investigation of Turbulent Channel Flow, J. Fluid Mech., 118 (1982), 341-377.

2. Viecelli, J. A., A Computing Method for Incompressible Flows Bounded by Walls, J. Comput. Phys., 8 (1971), 119-148.

3. Kato, C and Ikegawa, M., Large Eddy Simulation of Unsteady Turbulent Wake of a Circular Cylinder Using the Finite Element Method, ASME-FED, 117 (1991), 49-56.

4. Kato, C, Kaiho, M. and Manabe, A., An Overset Finite-Element Large-Eddy Simulation Method With Application to Turbomachinery and Aeroacoustics, Trans.ASME, 70 (2003), 32- 43.

5. Uddin, M. A., Kato, C., Yamade, Y., Ohshima, N., Tanahashi, M., and Miyauchi, T., "Large Eddy Simulation of Homogeneous Isotropic Turbulent Flow Using the Finite Element Method"- JSME Int. J, Ser. B, 49-(1), (2006), 102-114.

6. Vincent, A. and Meneguzzi, M., The Spatial Structure and Statistical Properties of Homogeneous Turbulence, J. Fluid Mech., 225 (1991), 1-20.

7. Jimenez, J., Wray, A. A., Saffman, P. G. and Rogallo, R. S., The Structure of Intense Vorticity in Isotropic Turbulence, J. Fluid Mech., 255 (1993), 65-90. 
8. Tanahashi, M., Miyauchi, T. and Ikeda, J., Scaling Law of Coherent Fine Scale Structure in Homogeneous Isotropic Turbulence, Proc. of the 11th Symposium on Turbulent Shear Flows., 1 (1997), 4-17-4-22.

9. Tanahashi, M., Uddin, M. A., Iwase, S. and Miyauchi, T., Three Dimensional Feature of Coherent Fine Scale Eddies in Homogeneous Isotropic Turbulence, Trans. Jpn. Soc. Mech. Eng., (in Japanese), 65-(638 B), (1999), 3237-3243.

10. Uddin, M. A., Tanahashi, M., Iwase, S. and Miyauchi, T., Visualization of Axes of Coherent Fine Scale Eddies in Homogeneous Isotropic Turbulence, Proc. of the 3rd Pacific Symposium on Flow Visualization and Image Processing (PSFVIP-3), CD-ROM Proc., C3-2, F3204 (2001), 1-7.

11. Sagaut, P., Large Eddy Simulation for Incompressible Flows, Springer-Verlag Heidelberg, (2001), 7.

12. Smagorinsky, J., General Circulation Experiments with the Primitive Equations. I: The basic experiment, Month. Weath. Rev., 91-(3), (1963), 99-165.

13. Germano, M., Piomelli, U., Moin., P and Cabot, W.H., A Dynamic Subgrid-scale Eddy Viscosity Model, Phys. Fluids., A 3-(7), (1991), 1760-1765.

14. Yoshizawa, A., Tsubokura, M, Kobayashi, T and Taniguchi, N, Modeling of the dynamic subgrid-sacle viscosity in large eddy simulation, Phys. Fluid, 8-(8), (1996), 2254-2256.

15. Leonard, A., Energy Cascade in Large Eddy Simulations of Turbulent Fluid Flows, Adv. in Geophys., A $18,(1974), 237-248$

16. Piomelli, U. High Reynolds Number Calculations Using the Dynamic Subgrid-scale Stress Model. Phys. Fluids, A 5-(6), (1993), 1484-1490.

17. Horiuti, K., A Proper Velocity Scale for Modeling Subgrid-scale Eddy Viscosities in Large Eddy Simulation, Phys. Fluids., A 5-(1), (1993), 146-157. 